\title{
Sleep disorders in patients with cancer
}

\author{
Ruben Guzman-Marin, $\mathrm{MD}, \mathrm{PhD},{ }^{a}$ and Alon Y Avidan, $\mathrm{MD}, \mathrm{MPH}^{\mathrm{b}}$ \\ ${ }^{a}$ Department of Neurology, Los Angeles Medical Center, Southern California Kaiser Permanente Medical Group; and ${ }^{\text {bUCLA Sleep }}$ \\ Disorders Center, Department of Neurology, David Geffen School of Medicine at UCLA, Los Angeles, California
}

\begin{abstract}
Sleep disturbances are common among patients with cancer for many reasons. Sleep problems can be present at any stage during treatment for cancer and in some patients, sleep disturbance may be the presenting symptoms that lead to the diagnosis of some types of cancer. Poor sleep impairs quality of life In people with cancer, but most do not specifically complain of sleep problems unless they are explicitly asked. Insomnia and fatigue are most common sleep disorders in this cohort, although primary sleep disorders, including obstructive sleep apnea and restless legs syndrome, which are common in the general population, have not been carefully studied in the oncology setting despite significant their impairment of quality of life.
\end{abstract}

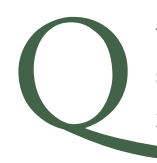
uality sleep is an essential element to tissue repair, proper immune function, and mental health. Chronic lack of sleep has been associated with depression, anxiety, and decreased cognitive function. ${ }^{1}$ In people with cancer, poor quality of sleep reduces quality of life, ${ }^{2}$ but unfortunately, most patients with cancer do not mention sleep problems unless explicitly asked. Sleep problems are common in this group of patients and may lead to a variety of sleep disturbances, which the International Classification of Sleep Disorders (ICSD III $)^{3}$ groups into 8 categories: Insomnia, Sleep-related breathing disorders, Central disorders of hypersomnolence, Parasomnias, Circadian rhythm sleep-wake disorders, Sleep-related movement disorders, Isolated symptoms, Apparent normal variants and unresolved issues, and Other sleep disorders

Most of the work addressing quality of life issues in patients with cancer has focused primarily on insomnia and fatigue, but both patients living with cancer and long-term survivors are at risk of having other sleep problems. ${ }^{4,5}$ Moreover, primary sleep disorders - including sleep-disordered breathing, such as obstructive sleep apnea, and motor disorders of sleep, such as restless legs syndrome (RLS) - have received little attention from the oncology community even though they are common in the general population. ${ }^{6,7}$ In this review, we present a conceptual framework on how sleep disorders may be present in patients with cancer, and the diagnostic features and management for the key sleep disturbances. (See online table of key studies that have reviewed the impact of cancer type on sleep.) Although most of the work related to sleep disorders in cancer patients has focused on insomnia and fatigue, there is a growing body of evidence confirming that other sleep disorders such as sleep disruption, insufficient sleep, restlessness, and diminished sleep duration are prevalent among patients with cancer as well as the general population, and that they decrease quality of life (QoL).

\section{Insomnia}

Insomnia is defined by the International Classification of sleep disorders (ICSD-3) ${ }^{3}$ as difficulty in initiating and/or maintaining sleep, or waking up too early, which are associated with chronically nonrestorative and poor-quality sleep. The sleep difficulty occurs despite an adequate opportunity and circumstances for sleep. Rates of insomnia are higher in patients with cancer, and it is estimated that as many as 30\%-50\% of people living with cancer experience sleep disturbance independent of the type of cancer (Table). ${ }^{8}$ Insomnia is perhaps the most common sleep problem among patients with cancer. In a recent study, rates of insomnia in patients with cancer were found to be nearly three times higher than the rates in the general population. ${ }^{9}$

Predisposing factors for insomnia in the general population include advanced age, race, female gender, personal or family history of insomnia, and a co-occurrence of a psychiatric disorder. ${ }^{8,10}$ Low socioeconomic status and educational level also are associated with insomnia. In the oncology setting, patients with breast and lung cancer have reported the existence of insomnia even before their diagnosis or initiation of adjuvant treatment. Patients with cancer experience multiple conditions associated with the disease, including pain, altered activity and rest patterns, altered hormone secretion, and cyto-

Accepted for publication March 2, 2015. Correspondence: Alon Y Avidan, MD, MPH; avidan@mednet.ucla.edu. Disclosures: Dr Guzman has no disclosures; Dr Avidan is on the speakers bureau for Merck and Xenoport. JCSO 2015;13:148-155. C2015 Frontline Medical Communications. DOI 10.12788/jcso.0126. 
kine production, that may affect sleeping and waking. ${ }^{11}$

Spielman's 3P model of insomnia is a heuristic device for assessing insomnia (Figure 1). ${ }^{12}$ The $3 \mathrm{P}$ model is a diathesis-stress model that includes predisposing, precipitating, and perpetuating factors. The predisposing factors can be physiological (eg, physiological hyperarousal, elevated cortisol), psychological (eg, tendency to worry), or social (eg, work schedule incompatible with sleep schedule). Precipitating factors, such as stressful life events (eg, cancer diagnosis) that trigger the acute onset of insomnia. Perpetuating factors, such as maladaptive coping skills or an extension of time in bed, can then contribute to the acute insomnia's developing into a chronic or longer-term disorder. Within this framework, predisposing factors represent a constant vulnerability for insomnia. Precipitating factors trigger acute insomnia, but the influence of these factors diminishes over time, whereas perpetuating factors then come into play and serve to maintain the insomnia. The perpetuating factors are typically the targets of treatment. ${ }^{12}$

Precipitating factors of insomnia are overpowering in patients with cancer. ${ }^{13}$ They include distressing cancerrelated symptoms such as pain, nausea, hot flashes, incontinence, diarrhea, delirium, draining lesions, pruritus, and respiratory compromise. ${ }^{13}$ Chemotherapy, surgery, biotherapy, radiation therapy, bone marrow transplantation, and associated hospitalizations, as well as several classes of medications such as steroids and opioids, can affect both circadian and homeostatic processes. Depression, anxiety, and worry often accompany a cancer diagnosis or terminal illness, contributing to insomnia. ${ }^{14,15}$

Patients with cancer are at particular risk for developing chronic insomnia. Health care providers routinely encourage them to get extra rest, conserve energy by decreasing normal daytime activities, and nap when tired, particularly during active treatment. ${ }^{13}$ Patients may not have the benefit of normal zeitgebers (environmental cues, such as sunlight) if they can perform activities only while in bed, lack social interaction, skip regular meals, or nap late in the day, which result in further alteration of the circadian rhythms. ${ }^{8,16}$

The assessment of insomnia in the clinical setting usually requires the use of self-reported measures. ${ }^{17}$ Several subjective tools for measuring sleep-wake disturbances have been validated in recent years for use in patients with cancer in clinical and research settings, among them, the Pittsburgh Sleep Quality Index, the Insomnia Severity Index, the Clinical Sleep Assessment for Adults, and keeping a daily diary of sleep perception for 2 weeks. Effective management of insomnia should prioritize the alleviation or control of cancer or treatment-related factors that are contributing to or perpetuating the disorder. ${ }^{18}$ Other comorbid medical and psychiatric conditions also must be identified and treated.

\section{Managing insomnia}

Benzodiazepines have been the primary medication for treating insomnia in both the general and the oncology populations along with antidepressants and antihistamines. There are a number of disadvantages in treating insomnia with benzodiazepines and, to a lesser extent, with the newer selective benzodiazepine receptor agonists such as zolpidem, zaleplon, eszopiclone, and a recently approved orexin receptor antagonist, suvorexant. Nonscheduled hypnotics include ramelteon, a melatonin receptor agonist and an ultra-low-dose doxepin. The benzodiazepines as a class may induce next-day sedation that can result in memory and cognitive impairment, falls due to psychomotor impairment, psychological and sometimes physiological dependence, and rebound insomnia. ${ }^{8,19-21}$ When life expectancy is short, medication is recommended as the treatment of choice for insomnia because the results are more rapid and because patients receiving palliative care tend to be less able to participate in dedicated cognitive behavioral therapy for insomnia (CBTi). ${ }^{15} \mathrm{CBT}$, which originated in the 1980 s, is an integration of cognitive therapy and behavioral modification techniques.

The choice of hypnotic may depend on the type of insomnia. If the major problem is falling asleep, then a shorthalf-life, fast-acting drug may be preferable. If the problem is related to frequent awakenings and sleep maintenance insomnia, then a longer-acting hypnotic might be more effective. Most hypnotics approved by the US Food and Drug Administration (FDA) are indicated for the treatment of sleep onset insomnia, whereas eszopiclone, zolpi-

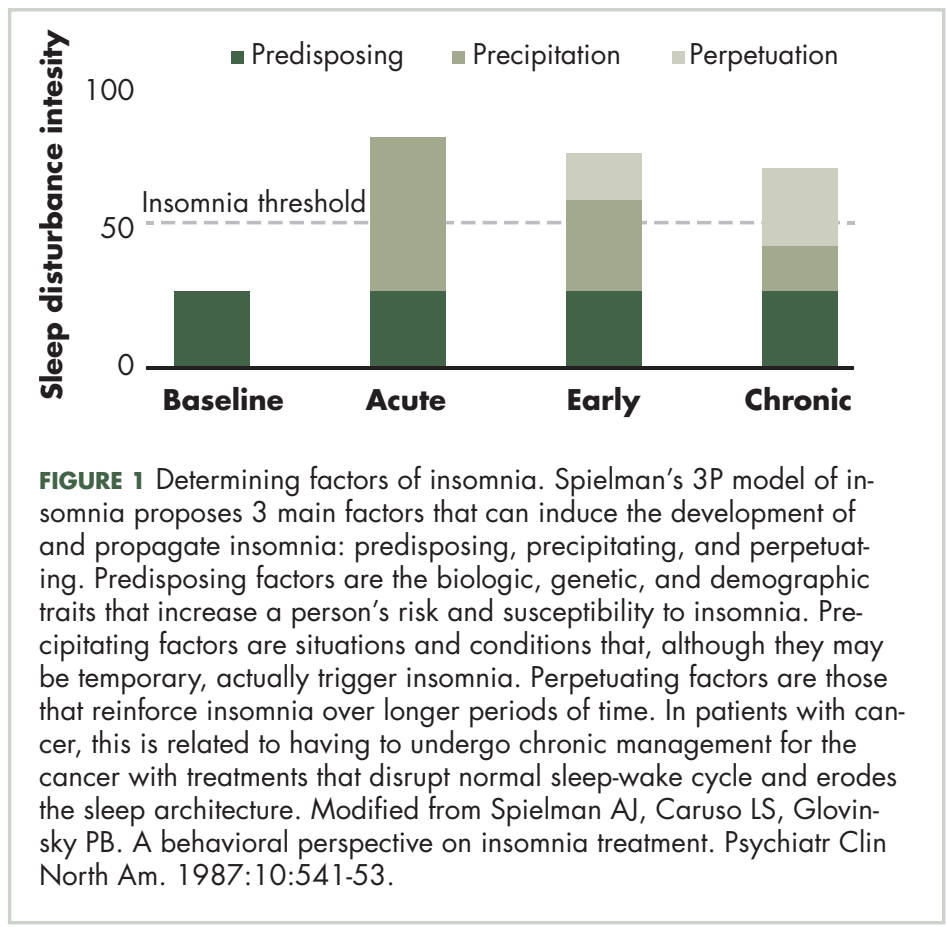


dem extended release, and suvorexant are also indicated for the treatment of sleep onset and sleep maintenance insomnia. An increasing body of research has emphasized that CBTi is as effective as medication for the short-term treatment of primary insomnia. ${ }^{8}$

\section{Fatigue}

Fatigue is a common symptom in patients with cancer and is nearly universal in those receiving cytotoxic chemotherapy, radiation therapy, bone-marrow transplantation, or treatment with biological response modifiers (Table). ${ }^{22}$ Fatigue is a common feature in other sleep disorders and is included here because of its common occurrence in patients with cancer and because its management may overlap with other sleep problems. Patients perceive fatigue to be the most distressing symptom associated with cancer and its treatment, more distressing even than pain or nausea and vomiting, which can generally be managed by medications in most patients. ${ }^{23}$ Fatigue is defined as the perception of unusual tiredness that varies in pattern or severity and has a negative impact on the ability to function. ${ }^{17}$ It is one of the most common and most severe symptoms in patients with solid tumors, including those with primary brain tumors. Cross-sectional studies have reported fatigue as the most prevalent symptom, with $40 \%-70 \%$ of patients with primary brain tumor reporting that they had fatigue throughout the disease trajectory. ${ }^{24,25}$ Although fatigue is prevalent throughout the trajectory, cranial radiation remains the most common and significant cause, with $80 \%$ of patients with primary brain tumor reporting fatigue during the course of radiation. ${ }^{25,26}$ In long-term survivors of cancer, the effects of fatigue may be long lasting. ${ }^{27}$ Fatigue has been reported to occur as early as the first week of treatment, the incidence and severity of fatigue markedly increases with cumulative radiation and often continues well into the postradiation period. These symptoms had a cyclical pattern, with increased severity on days 1-21 and again during days 30-35 after the start of radiation. This can occur regardless of the tumor grade, with one report indicating that $39 \%$ of patients with low-grade glioma reported severe fatigue several years after completion of therapy. ${ }^{28,29}$

The occurrence of fatigue beyond the acute phase of treatment was first noted in studies assessing the long-term quality of life of breast cancer survivors, which found that many women continued to experience feelings of fatigue, reductions in energy, and disruptions in activity level years after their diagnosis and treatment. ${ }^{4,5,30}$ The significance of the relationship between fatigue and altered sleep is supported by several reports that concluded that insomnia disorders added additional risk for experiencing intense and persistent fatigue even after cancer treatment concludes. ${ }^{8,31}$

\section{Managing fatigue}

In 2011, the National Comprehensive Cancer Network (NCCN) published the updated guidelines for the assessment and management of cancer-related fatigue. ${ }^{32} \mathrm{~A}$ detailed description of the recommendations for management of fatigue goes beyond the scope of this review, but a few general considerations are important to emphasize. For those cases in which patients report moderate to severe fatigue, the recommendations include evaluating the patient for contributing and treatable factors, including pain, emotional distress, anemia, altered sleep hygiene, nutritional deficiencies, and comorbid conditions, such as thyroid dysfunction. Once these have been evaluated and treatment initiated, if indicated, general recommended strategies to manage fatigue would include energy conservation techniques such as setting priorities, delegating activities that drain energy, structuring the daily routine, using labor-saving devices, and use of distraction. Naps should be limited to about 20 minutes to avoid interfering with the normal sleep-wake cycle and the risk of waking up groggy from deep sleep. ${ }^{32,33}$

The NCCN guidelines ${ }^{32}$ reviewed studies that examined pharmacologic treatment of fatigue, including psychostimulants, hemopoietic growth factors, antidepressants, and progestational steroids. Only psychostimulants (ie, methylphenidate) showed a small but significant improvement in fatigue. ${ }^{34,35}$ Other agents evaluated as part of the NCCN review did not have sufficient evidence to support use in patients with cancer-related fatigue, including antidepressants (no efficacy in solid tumors), corticosteroids (temporary responses only), L-carnitine (improvement only if subjects were deficient), and donepezil (no improvement in advanced cancer). More recently, an open-label randomized pilot trial of 4-week efficacy of methylphenidate and modafinil in 24 patients with brain tumors reported improvement in fatigue as measured by the Brief Fatigue Inventory (BFI) total score and Profile of Moods States fatigue subscale (POMS-F) instrument with either methylphenidate or modafinil, adding further support of the use of these agents in patients with primary brain tumors. ${ }^{36}$

\section{Sleep-disordered breathing}

Obstructive sleep apnea (OSA) is thought to affect about $5 \%$ of the adult population in the United States. ${ }^{37}$ Depending on how OSA is defined, estimates of its prevalence in adults are as high as $20 \% .{ }^{38}$ Age, gender, and body mass index all have an important impact on prevalence in various cohorts. In one study, apnea was present in $60 \%$ or more of individuals who experienced a stroke $\mathrm{e}^{39}$ and its prevalence is increased in the context of other chronic conditions, such as congestive heart failure, polycystic ovary syndrome, and asthma. Although OSA occurs in individuals of normal weight, its prevalence may be markedly underesti- 
mated in this population because of failure to consider this diagnosis in thin individuals.

Typical daytime symptoms of OSA include excessive daytime sleepiness, afternoon drowsiness, forgetfulness, impaired concentration and attention, personality changes, and morning headaches. The patient with upperairway resistance syndrome (UARS) typically complains of insomnia and daytime fatigue rather than sleepiness, myalgias (similar to those reported by fibromyalgia patients), migraine-like headaches, postural hypotension, and dizziness. Nocturnal signs and symptoms of OSA and UARS include snoring, snorting, observed apneas, awakening with a sensation of choking or gasping, unexplained tachycardia, restless sleep, sweating during sleep, nocturia, bruxism, and nocturnal gastroesophageal reflux. Insomnia, disrupted sleep, sleepwalking, sleep terrors, and confusional arousals are more commonly expressed by UARS patients. ${ }^{3}$

Recent data has highlighted a link between sleep apnea and cancer. ${ }^{40,41} \mathrm{~A}$ statistically significant trend for increasing cancer mortality was observed with higher levels of sleep apnea, measured either by the apnea-hypopnea index or degree of overnight hypoxemia. ${ }^{40}$ The study was underpowered to determine whether risk for cancer mortality differed among various types of cancer.

Sleep apnea is caused by upper airway collapse, but may be worsened by specific cancers (Figure 2). A high incidence of patients with head and neck cancer suffer from sleep apnea as a comorbidity or as a result of surgery or radiation therapy. ${ }^{42,43}$

Given that many patients presenting with cancer have many of the risk factors known to cause obstructive sleep apnea a detailed evaluation is recommended especially in those who present with complaints of snoring, difficulties with breathing at night, witnessed apneic episodes and excessive daytime sleepiness.

\section{Managing sleep-disordered breathing}

The approach to management of obstructive sleep apnea and hypopnea syndromes involves both general measures and interventions that address specific abnormalities. For most patients, nasal continuous positive airway pressure is the most effective medical therapy for control of sleep apnea.

General measures for identifying and addressing coexistent lifestyle issues that exacerbate OSA should be part of treatment of all patients. If sleep-disordered breathing is more prominent in the supine position, positional therapy to avoid sleep in the supine position is useful. Alcohol, hypnotic drugs, and other central nervous system depressant drugs interfere with the arousal response that terminates apneic episodes. Therefore, patients should avoid alcohol use and should not take hypnotics or sedatives. If a specific cause for upper-airway obstruction is found, an otorhino- laryngologic or maxillofacial evaluation is recommended for possible surgical intervention and trials of orthodontic devices, including tonsillectomy or adenoidectomy for enlarged tonsils or adenoids and correction of retrognathia or micrognathia. Results indicate that dental devices may be useful to those patients with mild to moderate sleep apnea with some degree of retrognathia or micrognathia. If chronic rhinitis is found, nasal steroid sprays may be beneficial.

\section{Restless legs syndrome}

One of the possible contributing factors in patients with insomnia, especially sleep initiation insomnia, is the presence of restless legs syndrome (RLS) or Willis-Ekbom disease. It is increasingly recognized as a common sensorimotor disorder that is characterized by uncomfortable and unpleasant sensations in the legs that may cause considerable sleep disruption and insomnia complaints. ${ }^{44}$

The prevalence of RLS in cancer patients undergoing chemotherapy is $18.3 \%$, about double of that expected in the general population. Its occurrence is much more frequent in female patients and with longer-term chemotherapy. Cancer patients who are afflicted by RLS have significantly higher levels of anxiety and depression and a poorer quality of life, especially in the physical well-being dimension.

Recent literature provides data on scenarios in which patients presenting with RLS symptoms, presented specifically because of iron deficiency in the setting of multiple myeloma, a hematologic malignancy characterized by clonal proliferation of plasma cells in the bone marrow and,

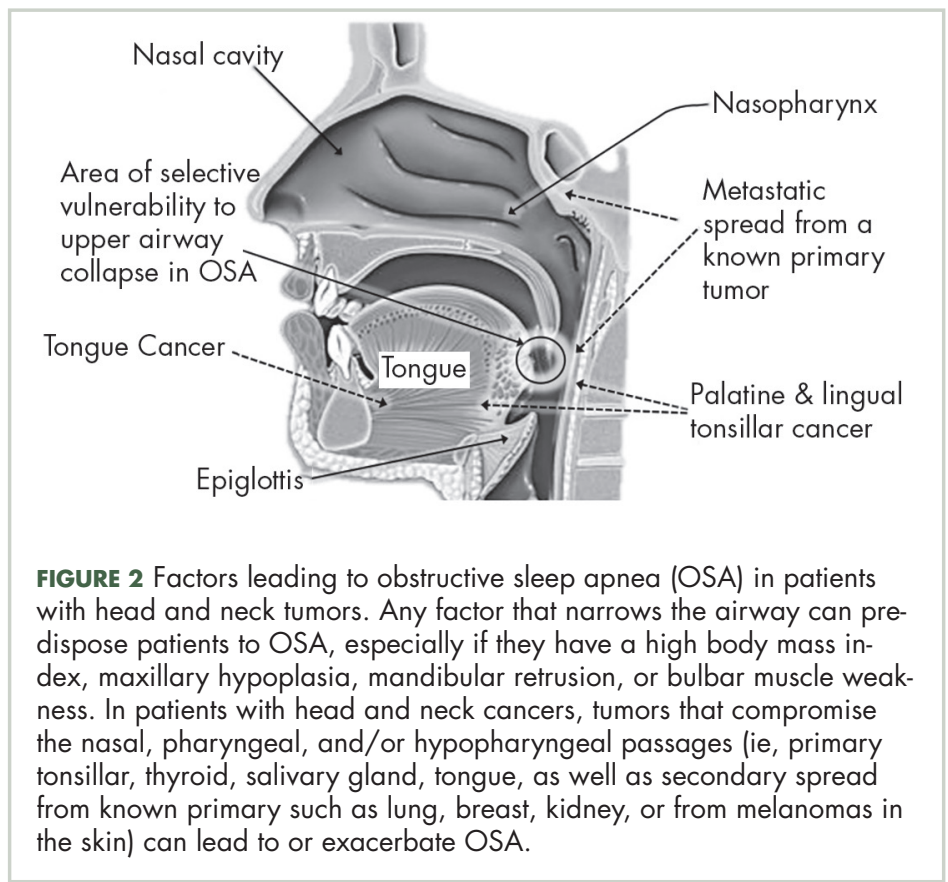


usually, the presence of a monoclonal immunoglobulin in the blood and/or urine. ${ }^{45}$

It is of note that anemia and reduced ferritin levels may be observed in cancer patients. Thus, further studies on the relationship between RLS and cancer should consider iron and ferritin levels. In healthy populations with RLS, a ferritin level of less than $50 \mathrm{ng} / \mathrm{mL}(50 \mu \mathrm{g} / \mathrm{L})$ signifies a potential role for iron deficiency in the disease and may require iron supplementation. In fact, the authors are aware of situations in which the discovery of low ferritin level in healthy adults who were evaluated for RLS, and subsequent causes of iron deficiency led to the diagnosis of colon cancer. Recognition and treatment of RLS in cancer patients is an important target in clinical management and may improve quality of life and overall health outcomes in these patients. ${ }^{46}$

\section{Managing RLS}

The 4 major classes of drugs that have been shown to be effective in the management of RLS are dopaminergic drugs, benzodiazepines, anticonvulsants, and opioids. The first approach for patients with symptoms consistent with RLS symptoms is to draw a serum ferritin level. Patients with levels less than $50 \mathrm{ng} / \mathrm{mL}(50 \mu \mathrm{g} / \mathrm{L})$ should begin iron replacement therapy with iron sulfate along with vitamin $\mathrm{C}$ to improve absorption.

When iron stores are normal, nonergotamine dopamine $\left(\mathrm{D}_{2} \mathrm{D} 3\right)$ agonists such as ropinirole, pramipexole, rotigotine, may be started. Dopamine agonists are preferred because they have been approved by the FDA specifically for the treatment of RLS. Major side-effects include unpredictable sleep attached, gastrointestinal side effects, postural orthostatic hypotension, and at higher doses, rare cases of impulse-control behaviors such as compulsive gambling. The FDA recently approved gabapentin enacarbil, an alpha 2-delta ligand for the treatment of moderate to severe primary RLS in adults.

\section{Excessive daytime sleepiness}

Hypersomnia or excessive daytime sleepiness (EDS) symptoms are essential features of distinct entities that include behaviorally induced insufficient sleep; disorders in circadian rhythm; narcolepsy; and idiopathic, recurrent, and other hypersomnias (eg, caused by a CNS-acting drug or an underlying co morbid medical condition). The severity, timing, and duration of sleepiness are important clues to understand the underpinnings of sleepiness as a symptom. As mentioned earlier EDS may be part of the symptoms associated with insomnia and sleep apnea, therefore the history provided by the patients help to elucidate whether excessive daytime sleepiness is the primary symptom or is associated with another sleep disorder.

Daytime sleepiness and sleep duration are associated with poor functional status, quality of life, and mortality in the general population. In a recent study cancer survivors were more likely than control to report excessive daytime sleepiness as assessed by the Epworth Sleepiness Scale (ESS). Disturbances in daytime sleepiness and sleep duration persist among long-term cancer survivors and should be monitored in routine survivorship care. ${ }^{47}$ Daytime sleepiness seems to be common in patients undergoing treatment for brain tumors. ${ }^{48}$ Adult survivors of childhood brain tumors, treatment with a radiation at a younger age at treatment and those children with craniopharyngioma report significant problems with sleep as adults. ${ }^{49}$

In some instances, EDS may be part of the presenting symptom in some paraneoplastic neurological disorders (PND). A recently discovered symptomatic variant of narcolepsy with cataplexy occurred in patients with anti-Ma2encephalitis, a common autoimmune disorder. ${ }^{50}$ About $3 \%-5 \%$ of patients with small-cell lung cancer ${ }^{51} 15 \%-$ $20 \%$ with thymomas, and 3\%-10\% with B-cell or plasmacell neoplasms develop PND. The prevalence of PND in other neoplasms, including breast or ovary cancer and others cancers, is well less than $1 \% \cdot{ }^{51}$ Cancer and associated paraneoplastic syndromes can severely affect circadian processes through altered hormone secretion (ie, cortisol and melatonin) and cytokine production.

\section{Managing EDS}

The management of EDS focuses on the control of this symptom to allow optimal social and professional productivity by maintaining the patient's alertness throughout the day. The drugs that are commonly used to manage EDS and sleep attacks are the nonamphetamine stimulants (known as wake-promoting agents) such as armodafinil and modafinil, and CNS stimulants such as methylphenidate and dextroamphetamine. Nonpharmacologic treatments, include short "power" naps and support therapy, should also be emphasized. Short naps of 15-20 minutes 3 times during the day help maintain alertness and have been shown to have a recuperative power in management of EDS.

\section{Rapid eye movement sleep behavior disorder}

Rapid eye movement (REM) sleep behavior disorder (RBD) is a parasomnia characterized by vigorous dream enacting behaviors associated with nightmares and abnormally increased electromyographic activity during REM sleep. ${ }^{3}$ It is thought that excessive electromyographic (EMG) activity during REM sleep in RBD patients reflects dysfunction of the brainstem structures that are responsible for muscle atonia during REM sleep. ${ }^{52,53} \mathrm{RBD}$ is very common in several neurodegenerative diseases, including multiple system atrophy, dementia with Lewy bodies and Parkinson's disease. 
RBD has been documented to be the presenting symptom in a patient with brainstem lymphoma, ${ }^{54}$ and in a patient with tonsillar carcinoma..$^{55} \mathrm{RBD}$ has also been documented to be present in patients with advanced gastric and renal carcinomas. ${ }^{56}$ The case of the patient with tonsillar carcinoma seemed to be associated with a paraneoplastic syndrome..$^{55}$

\section{Managing RBD}

Safety intervention by removing sharp objects from the bedroom area is always a good first step to protect patients from hurting themselves during violent dream enchantment. The most commonly prescribed drug therapy for $\mathrm{RBD}$ is clonazepam at a dosage of $0.5-1.0 \mathrm{mg}$ at bedtime. Melatonin at high doses (3-12 mg) has also been shown to control the symptoms in mild cases effectively. ${ }^{57}$

\section{Circadian rhythms sleep disorders}

Circadian rhythms are endogenous rhythms with a periodicity of about 24 hours. These are synchronized to the environment by social and work schedules by light and nonlight stimuli. The circadian rhythms sleep disorders (CRSDs) generally result from a misalignment between the timing of the circadian rhythm and the external environment (eg, shift work) or a dysfunction of the circadian clock or its afferent and efferent pathways (eg, delayed sleep phase syndrome). The most common symptoms of these disorders are difficulties with sleep onset and/or maintenance and excessive daytime sleepiness associated with impaired social and/or occupational performance. ${ }^{3}$

There has been an increasing interest in the possible role of environmental factors in the etiology of cancer. Of particular interest is the potential influence of exposure to light-at-night, and sleep disruption, on endocrine function and the regulation of hormones that are important in the etiology of some types of cancer. Persons who engage in night shift work are subject to the influence of both factors, and may exhibit altered hormone profiles as a result that could increase the risk of hormone-related diseases, including breast and prostate cancer. ${ }^{58}$

Light exposure during night shift work affects the circadian system of shift workers in several unfavorable ways, including by desynchronizing their 24-hour bodily rhythms and suppressing nocturnal melatonin secretion. ${ }^{59}$ Melatonin, a hormone closely linked to the circadian system, regulates the sleep-wake cycle in humans and has antimutagenic and oncostatic properties. ${ }^{60}$ Lower melatonin levels ${ }^{61}$ and sleep disruption ${ }^{62}$ have been linked to increased risk for cancer.

Dysregulation of circadian physiology is often prominent and extensive in patients with advanced cancer. Disrupted circadian cycles are evident in endocrine, immune, metabolic, and cellular systems. ${ }^{63-65}$ It has been suggested that dysruption of circadian hypothalamic-pituitary-adrenal (HPA) rhythms has been linked specifically with advancing cancer and accelerated tumor growth rates. ${ }^{66-68}$ Moreover, flattening of the diurnal cortisol rhythm predicts early lung cancer death and there is growing evidence that circadian disruption accelerates tumor progression. ${ }^{67}$

\section{Managing circadian rhythm sleep disorders}

The management for most of the CRSDs requires a multimodal approach to accelerate circadian realignment with timed exposure to light. This includes avoidance of bright light at inappropriate times and adherence to scheduled sleep and wake times. In addition, pharmacologic agents are recommended for some of the CRSDs. For example in the case of delayed sleep phase syndrome, and shift work disorders, timed low dose melatonin can help to advance circadian rhythms; in the case of shift work disorder, wake enhancing agents such as caffeine, modafinil and armodafinil are options for the management of excessive daytime sleepiness.

Timed bright light therapy and avoidance of light at the wrong time of the day can help accelerate and maintain entrainment to the shift schedule. For night workers, circadian rhythms need to be delayed, so that the highest sleep propensity occurs during the day, rather than at night. Intermittent bright light exposure $(20 \mathrm{~min} / \mathrm{h})$ and avoidance of bright light exposure in the morning during the commute home (using driving-safe sunglasses) has being shown to accelerate circadian adaptation to night shift.

\section{Conclusion}

In patients with cancer, fatigue and insomnia seem to be the most pervasive sleep disturbances. A new paradigm shift in the management of patients with cancer who complain of poor sleep is to screen effectively for the underlying disturbances, assess their contribution to next-day function, and advocate for specific treatment options to improve quality of life.

\section{References}

1. Ehlers CL, Frank E, Kupfer DJ. Social zeitgebers and biological rhythms: a unified approach to understanding the etiology of depression. Arch Gen Psych. 1988;45:948.

2. Davidson JR, MacLean AW, Brundage MD, Schulze K. Sleep disturbance in cancer patients. Soc Sci Med. 2002;54:1309-1321.

3. American Academy of Sleep Medicine. International classification of sleep disorders, 3rd ed. Darien, IL: American Academy of Sleep Medicine, 2014.

4. Berglund G, Bolund C, Fornander T, Rutqvist LE, Sjödén P-O. Late effects of adjuvant chemotherapy and postoperative radiotherapy on quality of life among breast cancer patients. Eur J Cancer Clin Oncol. 1991;27:1075-1081.

5. Dow KH, Ferrell BR, Leigh S, Ly J, Gulasekaram P. An evaluation of the quality of life among long-term survivors of breast cancer. Breast Cancer Res Treat. 1996;39:261-273.

6. Young T, Palta M, Dempsey J, Skatrud J, Weber S, Badr S. The occurrence of sleep-disordered breathing among middle-aged adults. $\mathrm{N}$ Eng1 J Med. 1993;328:1230-1235. 
7. Karatas M. Restless legs syndrome and periodic limb movements during sleep: diagnosis and treatment. Neurologist. 2007;13:294-301.

8. Savard J, Morin CM. Insomnia in the context of cancer: a review of a neglected problem. J Clin Oncol. 2001;19:895-908.

9. Palesh OG, Roscoe JA, Mustian KM, et al. Prevalence, demographics, and psychological associations of sleep disruption in patients with cancer: University of Rochester Cancer Center-Community Clinical Oncology Program. J Clin Oncol. 2010;28:292-298.

10. Vena C, Parker K, Cunningham M, Clark J, McMillan S. Sleep-wake disturbances in people with cancer part I: an overview of sleep, sleep regulation, and effects of disease and treatment. Oncol Nurs Forum. 2004;13;31:747-71

11. Clark J, Cunningham M, McMillan S, Vena C, Parker K. Sleep-wake disturbances in people with cancer part II: evaluating the evidence for clinical decision making. Oncol Nurs Forum. 2004; 13;31:74771

12. Spielman AJ, Caruso LS, Glovinsky PB. A behavioral perspective on insomnia treatment. Psychiatr Clin North Am. 1987:10:541-53.

13. Woodward SC. Cognitive-behavioral therapy for insomnia in patients with cancer. Clin J Oncol Nurs. 2011;15:E42-E52.

14. Graci G. Pathogenesis and management of cancer-related insomnia. J Support Oncol. 2005;3:349-359.

15. Kvale EA, Shuster JL. Sleep disturbance in supportive care of cancer: a review. J Palliat Med. 2006;9:437-450.

16. Parker KP, Bliwise DL, Ribeiro M, et al. Sleep/wake patterns of individuals with advanced cancer measured by ambulatory polysomnography. J Clin Oncol. 2008;26:2464-2472.

17. Barsevick AM, Cleeland CS, Manning DC, et al. ASCPRO recommendations for the assessment of fatigue as an outcome in clinical trials. J Pain Symptom Manage. 2010;39:1086-1099.

18. O'Donnell JF. Insomnia in cancer patients. Clin Cornerstone. 2004;6:S6-S14

19. Hu D-S, Silberfarb P. Management of sleep problems in cancer patients. Oncology (Williston Park, NY). 1991;5:23.

20. Griffiths RR, Johnson MW. Relative abuse liability of hypnotic drugs: a conceptual framework and algorithm for differentiating among compounds. J Clin Psych. 2005;66(suppl 9):31-41.

21. Doghramji PP. Trends in the pharmacologic management of insomnia. J Clin Psych. 2006;67:5-8

22. Berger AM. Update on the state of the science: sleep-wake disturbances in adult patients with cancer. Oncol Nurs Forum. 2009;36:E165-77

23. Berger AM, Abernethy AP, Atkinson A, et al. Cancer-related fatigue. J Natl Compr Canc Netw. 2010;8:904-931.

24. Armstrong TS, Cron SG, Bolanos EV, Gilbert MR, Kang DH. Risk factors for fatigue severity in primary brain tumor patients. Cancer. 2010;116:2707-2715.

25. Lovely MP, Miaskowski C, Dodd M. Relationship between fatigue and quality of life in patients with glioblastoma multiformae. Oncol Nurs Forum. 1999;26:921-25.

26. Miaskowski C. Gender differences in pain, fatigue, and depression in patients with cancer. JNCI Monographs. 2004;32:139-143.

27. Manley PE, McKendrick K, McGillicudy M, et al. Sleep dysfunction in long term survivors of craniopharyngioma. J Neurooncol. 2012;108:543-549.

28. Drappatz J, Schiff D, Kesari S, Norden AD, Wen PY. Medical management of brain tumor patients. Neurol Clin. 2007;25:1035-1071.

29. Struik K, Klein M, Heimans JJ, et al. Fatigue in low-grade glioma. J Neurooncol. 2009;92:73-78.

30. Beisecker AE, Cook MR, Ashworth J, et al. Side effects of adjuvant chemotherapy: perceptions of node-negative breast cancer patients. Psycho-oncology. 1997;6:85-93.

31. Mendoza TR, Wang XS, Cleeland CS, et al. The rapid assessment of fatigue severity in cancer patients. Cancer. 1999;85:1186-1196.

32. Guidelines NNCCN. 2012 Cancer-Related Fatigue. In: NCCN, ed. Fort Washington, PA2011.

33. Hayashi M, Watanabe M, Hori T. The effects of a 20 min nap in the mid-afternoon on mood, performance and EEG activity. Clin Neurophysiol. 1999;110:272-279.

34. Moraska AR, Sood A, Dakhil SR, et al. Phase III, randomized, double-blind, placebo-controlled study of long-acting methylphenidate for cancer-related fatigue: North Central Cancer Treatment Group NCCTG-N05C7 trial. J Clin Oncol. 2010;28:3673-3679.

35. Minton O, Richardson A, Sharpe M, Hotopf M, Stone PC. Psychostimulants for the management of cancer-related fatigue: a systematic review and meta-analysis. J Pain Symptom Manage. 2011;41:761767.

36. Gehring K, Patwardhan S, Collins R, et al. A randomized trial on the efficacy of methylphenidate and modafinil for improving cognitive functioning and symptoms in patients with a primary brain tumor. J Neurooncol. 2012;107:165-174.

37. Caples SM, Gami AS, Somers VK. Obstructive sleep apnea. Ann Intern Med. 2005;142:187-197.

38. Young T, Skatrud J, Peppard PE. Risk factors for obstructive sleep apnea in adults. JAMA. 2004;291:2013-2016.

39. Bassetti C, Aldrich MS. Sleep apnea in acute cerebrovascular diseases: final report on 128 patients. Sleep. 1999;22:217-224.

40. Nieto FJ, Peppard PE, Young T, Finn L, Hla KM, Farré R. Sleepdisordered breathing and cancer mortality: results from the Wisconsin Sleep Cohort Study. Am J Respir Crit Care Med. 2012;186:190194.

41. Campos-Rodriguez F, Martinez-Garcia MA, Martinez M, et al. Association between obstructive sleep apnea and cancer incidence in a large multicenter Spanish cohort. Am J Respir Crit Care Med. 2013;187:99-105.

42. Friedman M, Landsberg R, Pryor S, Syed Z, Ibrahim H, Caldarelli $\mathrm{DD}$. The occurrence of sleep-disordered breathing among patients with head and neck cancer. Laryngoscope. 2001;111:1917-1919.

43. Rombaux P, Hamoir M, Plouin-Gaudon I, Liistro G, Aubert G Rodenstein D. Obstructive sleep apnea syndrome after reconstructive laryngectomy for glottic carcinoma. Eur Arch Otorhinolaryngol. 2000;257:502-506.

44. Broman JE, Mallon L, Hetta J. Restless legs syndrome and its relationship with insomnia symptoms and daytime distress: epidemiological survey in Sweden. Psychiatry Clin Neurosci. 2008;62:472475

45. Arico D, Raggi A, Siragusa M, Zucconi M, Ferri R. Restless legs syndrome as the presenting symptom of multiple myeloma. J Clin Sleep Med. 2012;9:383-385.

46. Ostacoli L, Saini A, Ferini-Strambi L, et al. Restless legs syndrome and its relationship with anxiety, depression, and quality of life in cancer patients undergoing chemotherapy. Qual Life Res. 2010;19:531-537

47. Forsythe LP, Helzlsouer KJ, MacDonald R, Gallicchio L. Daytime sleepiness and sleep duration in long-term cancer survivors and noncancer controls: results from a registry-based survey study. Support Care Cancer. 2012;20:2425-2432.

48. Armstrong TS, Gilbert MR. Practical strategies for management of fatigue and sleep disorders in people with brain tumors. Neuro Oncol. 2012; 14:iv65-iv72.

49. Gapstur R, Gross CR, Ness K. Factors associated with sleep-wake disturbances in child and adult survivors of pediatric brain tumors: a review. Oncol Nurs Forum. 2009;36:723-31.

50. Rosenfeld MR, Eichen JG, Wade DF, Posner JB, Dalmau J. Molecular and clinical diversity in paraneoplastic immunity to Ma proteins. Ann Neurol. 2001;50:339-348.

51. Elrington G, Murray N, Spiro S, Newsom-Davis J. Neurological paraneoplastic syndromes in patients with small cell lung cancer. A prospective survey of 150 patients. Neurol Neurosurg Psychiatry. 1991;54:764-767.

52. Mahowald MW, Ettinger MG. Things that go bump in the night: the parasomnias revisited. J Clin Neurophysiol. 1990;7:119-144.

53. Boeve B, Silber M, Saper C, et al. Pathophysiology of REM sleep behaviour disorder and relevance to neurodegenerative disease. Brain. 2007;130:2770-2788.

54. Jianhua C, Xiuqin L, Quancai C, Heyang S, Yan H. Rapid eye movement sleep behavior disorder in a patient with brainstem lymphoma. Intern Med. 2012;52:617-621

55. Adams C, McKeon A, Silber MH, Kumar R. Narcolepsy, REM sleep behavior disorder, and supranuclear gaze palsy associated with Ma1 and Ma2 antibodies and tonsillar carcinoma. Arch Neurol. 2011;68:521-524. 
56. Shinno H, Kamei M, Maegawa T, et al. Three patients with cancer who developed rapid-eye-movement sleep behavior disorder. J Pain Symptom Manage. 2010;40:449-452.

57. Standards of Practice Committee, Aurora RN, Zak RS, et al. Best practice guide for the treatment of REM sleep behavior disorder (RBD). J Clin Sleep Med. 2010;6:85-95.

58. Davis S, Mirick DK. Circadian disruption, shift work and the risk of cancer: a summary of the evidence and studies in Seattle. Cancer Causes Control. 2006;17:539-545.

59. Arendt J. Shift work: coping with the biological clock. Occup Med. 2010;60:10-20.

60. Thomas CR, Reiter RJ, Herman TS. Melatonin: from basic research to cancer treatment clinics. J Clin Oncol. 2002;20:2575-2601.

61. Schernhammer ES, Berrino F, Krogh V, et al. Urinary 6-Sulphatoxymelatonin levels and risk of breast cancer in premenopausal women: the ORDET cohort. Cancer Epidemiol Biomarkers Prev. 2010;19:729-737.

62. Gallicchio L, Kalesan B. Sleep duration and mortality: a systematic review and meta-analysis. J Sleep Res. 2009;18:148-158.

63. Mazzoccoli G, Vendemiale G, De Cata A, Carughi S, Tarquini R.

Altered time structure of neuro-endocrine-immune system function in lung cancer patients. BMC Cancer. 2010;10:314

64. Hrushesky WJ, Grutsch J, Wood P, et al. Circadian clock manipulation for cancer prevention and control and the relief of cancer symptoms. Integr Cancer Ther. 2009;8:387-397.

65. Mormont M-C, Waterhouse J, Bleuzen P, et al. Marked 24-h rest/ activity rhythms are associated with better quality of life, better response, and longer survival in patients with metastatic colorectal cancer and good performance status. Clin Cancer Res. 2000;6:30383045.

66. Mormont M, Levi F. Circadian-system alterations during cancer processes: A review. Int J Cancer. 1997;70:241-247.

67. Sephton S, Spiegel D. Circadian disruption in cancer: a neuroendocrine-immune pathway from stress to disease? Brain Behav Immun. 2003;17:321-328.

68. Eismann EA, Lush E, Sephton SE. Circadian effects in cancer-relevant psychoneuroendocrine and immune pathways. Psychoneuroendocrinology. 2010;35:963-976. 